\title{
Effects of Acute Tryptophan Depletion on Mood and Facial Emotion Perception Related Brain Activation and Performance in Healthy Women with and without a Family History of Depression
}

\author{
Frederik M van der Veen*,', Elisabeth AT Evers ${ }^{2}$, Nicolaas EP Deutz ${ }^{3}$ and Jeroen AJ Schmitt ${ }^{4}$ \\ 'Department of Psychiatry, Erasmus Medical Centre, Rotterdam, The Netherlands; ${ }^{2}$ Department of Psychiatry and Neuropsychology, University \\ of Maastricht, Maastricht, The Netherlands; ${ }^{3}$ Department of Surgery, University of Maastricht, Maastricht, The Netherlands; ${ }^{4}$ Department \\ Nutrition and Health, Nestle Research Centre, Lausanne, Switzerland
}

\begin{abstract}
The present study examined the effects of acute tryptophan (Trp) depletion (ATD), a well-recognized method to lower central serotonin (5-HT) metabolism, on brain activation during a facial emotion perception task. Brain activation was measured using fMRI, and healthy female volunteers with a positive family history of unipolar depression $(\mathrm{FH}+)$ were compared to healthy female volunteers without such a history $(\mathrm{FH}-$ ). Participants viewed two morphed faces and were instructed to choose between the faces based either on the intensity of the emotional expression (direct task) or the gender of the face (incidental task). In the FH + group, depletion led to the expected lowering of mood, which partly determined the effect of depletion on performance and brain activation. A stronger mood lowering effect was associated with less accurate performance on faces expressing a negative emotion in the incidental task and a stronger right amygdala response to fearful faces in comparison to happy faces. These results were explained in terms of a mood-induced bias leading to a stronger impact of the expressed negative emotion which subsequently leads to more interference in the incidental task and a stronger amygdala response. It was concluded that the effects of ATD on mood, performance, and brain activation in a facial emotion perception task depend on family history of depression. Performance and brain activation partly depend on the effect of ATD on mood.
\end{abstract}

Neuropsychopharmacology (2007) 32, 2 16-224. doi: I 0. I038/sj.npp. I 301212; published online 4 October 2006

Keywords: serotonin; family history; depression; functional imaging; amygdala; facial emotions

\section{INTRODUCTION}

The neurotransmitter serotonin (5-HT) has long been implicated in mood regulation and affective disorders. A direct role of a serotonergic deficiency in depression remains to be established (Lacasse and Leo, 2005), but central and peripheral markers of lowered 5-HT function have been observed in depressed patients, including low plasma tryptophan (Trp) levels, reduced platelet 5-HT uptake, decreased cerebrospinal fluid 5-hydroxyindoleacetic acid levels, and altered 5-HT receptor and 5-HT transporter binding, although not always consistently (see Schatzberg et al, 2002; Maes and Meltzer, 1995).

\footnotetext{
*Correspondence: Dr FM van der Veen, Department of Psychiatry, Erasmus Medical Centre, PO Box 2040, 3000 CA Rotterdam, The Netherlands, Tel: + 3 I I04632336, Fax: + 3 I I04635867,

E-mail: f.vanderveen@erasmusmc.nl

Received 13 March 2006; revised and accepted 8 July 2006

Online publication: 31 August 2006 at http://www.acnp.org/citations/ Npp083106060167/default.pdf
}

One way to directly study the effects of $5-\mathrm{HT}$ on mood and cognition is by means of acute Trp depletion (ATD). During an ATD session, volunteers ingest a Trp-depleted but otherwise balanced amino-acid mixture. The ingestion of this mixture causes a sharp drop in Trp plasma levels, which leads to a reduced availability of $\operatorname{Trp}$ in the brain. Trp is an essential amino acid and is the precursor of 5-HT and consequently a drop in central Trp levels leads to a drop in central 5-HT metabolism (eg Carpenter et al, 1998).

The studies using ATD have reported a highly variable effect on mood of healthy volunteers, which appears to be dependent on individual characteristics. The majority of studies have found that healthy volunteers with no personal or family history of affective disorder and without specific genetic risk factors do not experience a mood lowering effect of ATD (eg Schmitt et al, 2000; Evers et al, 2005a,b). On the other hand, healthy volunteers with a family history of affective disorder (eg Benkelfat et al, 1994; Neumeister et al, 2002), patients with remitted depression (eg Booij et al, 2002; Neumeister et al, 2004), and healthy volunteers with specific genetic risk factors like carriers of the short 
allele variant of a polymorphism in the promoter region of the 5-HT transporter gene (Neumeister et al, 2002) generally do experience mood lowering effects of ATD.

The results with respect to the effects of ATD on emotional behavior have also been somewhat variable. However, it seems to be clear that 5-HT plays a crucial role in emotional behavior (for a review, see Lucki, 1998). An important aspect of human emotional behavior is the ability to recognize emotions in other individuals, which is especially important in social interaction. One way to recognize these emotions is to monitor facial expressions, which appears to be disturbed in depression (Bhagwagar et al, 2004; Gur et al, 1992; Surguladze et al, 2004). Gur et al found that depressed patients showed an overall stronger negative bias for recognizing emotional expressions and performed more poorly on discriminating happy and sad faces from neutral faces. On the other hand, Bhagwagar et al found that female volunteers whose depression was in remission showed enhanced recognition of fearful expressions. Finally, Surguladze et al (2004) showed that depressed patients had a stronger negative bias in categorizing facial expressions. In a number of studies, the effect of ATD on recognition of emotional face expressions has been studied, but the results of these studies are inconclusive. Harmer et al (2003) found that ATD decreased the recognition of fearful expressions in female volunteers, but not in males. On the other hand, Hayward et al (2005) reported that ATD enhanced the recognition of happy faces in a direct task for both sexes. Finally, Cools et al (2005) found that ATD did not affect recognition of facial expressions, but increased the blood oxygenation-level-dependent (BOLD) response at the border of the right amygdala and hippocampus in a contrast comparing fearful and happy facial expressions, but only in participants with high threat sensitivity scores as measured by the Behavioral Inhibition Scale (BIS, Carver and White, 1994).

The variability in effects of ATD on recognition of emotional facial expressions might be related to the variability in effects on mood, and in this way to differences in vulnerability of participants to changes in central 5-HT levels. To test this hypothesis, we designed a study in which we examined the effects of ATD on behavior and brain activation accompanying the direct and incidental perception of emotional face expressions. We compared a group consisting of healthy female volunteers with a positive family history of unipolar depression $(\mathrm{FH}+)$ with a matched group of healthy female volunteers without such a history $(\mathrm{FH}-)$. We hypothesized that the $\mathrm{FH}+$ group would experience the mood lowering effect of ATD (Benkelfat et al, 1994; Klaassen et al, 1999; Neumeister et al, 2002), but not the FH - group (eg Schmitt et al, 2000; Evers et al, 2005a,b). Both groups performed a facial emotion perception task which was developed by Winston et al (2003). In this task, pairs of morphed faces with intense or weak emotional expressions, and higher or lower percentages of a male face were judged on the intensity of the expression (direct task), or the gender of the face (incidental task). Winston et al (2003) found amygdala and fusiform gyrus responses independent of task instruction for more intense expressions. Right superior temporal sulcus was especially active in the direct task with more intense expressions. Finally, enhanced ventromedial prefrontal and somatosensory cortex activation was found in the direct task. Due to the inclusion of only female participants, it was hypothesized that ATD would decrease recognition of fearful faces as was found in the female volunteers of the study of Harmer et al (2003). Furthermore, it was expected that ATD would enhance the amygdala response to fearful faces, but that this effect would be dependent on threat sensitivity (Cools et al, 2005). Due to the expected stronger mood response in the $\mathrm{FH}+$ group, we expected this group to show exaggerated brain responses in emotion-related areas, like the amygdala. Finally, we tested the hypothesis that both the effects on performance and brain activation would be modulated by the effect of ATD on mood.

\section{METHODS}

\section{Participants}

A total of 14 volunteers with a family history of unipolar depression $(\mathrm{FH}+)$, and 19 healthy volunteers without a family history of depression $(\mathrm{FH}-)$ were recruited by advertisements in local papers and at the Maastricht University campus site. Family history of unipolar depression was defined as having at least one first-degree relative (father or mother, or brother or sister) who suffered from at least one confirmed episode of unipolar depression. The diagnosis was confirmed by their general health practitioner or psychiatrist. All participants were female and both groups were matched for age and educational level, which was measured on an eight-point scale. The participants' health was checked with an extensive medical questionnaire, which was evaluated by a medical doctor. Volunteers with a history of neurological or psychiatric disorders, other important health problems, excessive alcohol or drug use, present or past use of antidepressants or MDMA (ecstasy), and metal implants or other contra-indications for fMRI were excluded from this study. The ethical committee of University of Maastricht approved the study. All participants gave written informed consent and were paid 75 euro for their voluntary participation. In total, 13 participants belonging to the $\mathrm{FH}+$ group and 11 participants belonging to the $\mathrm{FH}$ - group were successfully scanned and had complete data. Age (mean \pm SEM: $21.4 \pm 0.6$ in the $\mathrm{FH}-$ group vs $23.8 \pm 1.6$ in the $\mathrm{FH}+$ group; $p=0.2$ ) and educational level (mean \pm SEM: $7.0 \pm 0$ in the $\mathrm{FH}-$ group vs $6.5 \pm 0.3$ in the $\mathrm{FH}+$ group; $p=0.2$ ) did not differ between the groups. Due to logistical problems, it was not possible to control for phase in the menstrual cycle. However, we did monitor menstrual phase, and post hoc tests showed that menstrual phase did not differ between groups $(p>0.5)$ and condition $(p>0.5)$.

\section{Stimuli}

Detailed description of the task and stimuli can be found in Winston et al (2003). Participants viewed pairs of morphed faces and were asked to assess which face expressed the strongest emotion (direct task) or was most masculine (incidental task). The faces were morphed between a neutral face of a male or female and a face with an emotional (fear, disgust, happiness, sadness) expression of a person of the opposite sex, using commercially available morphing software (Morpher 2.0). Eight different faces were 
used in the present experiment. These faces were selected from the Ekman and Friesen (1978) series of emotional faces. The presented pairs of faces consisted either of two more emotional expressions (morphed face contains 70 or $90 \%$ of the face with the emotional expression; high intensity) or more neutral expressions ( 10 or $30 \%$ of the face with the emotional expression; low intensity). The two faces that were presented at the same time always expressed the same emotion and had the same identity; only the intensity of the expression and the percentage of one or the other identity differed. The pairs of faces were presented for a maximum of $2500 \mathrm{~ms}$, but disappeared after a response was given (Inter-stimulus interval was $3000 \mathrm{~ms}$ ). The stimuli were presented in blocks of 10, which consisted of eight different face pairs (the four different emotions at two intensity levels) randomly mixed with two null events. Each block was preceded by a short instruction, and the two types of tasks were sequentially presented.

\section{Procedure}

The experiment was conducted as a placebo-controlled, double-blind, crossover design. Participants were tested on two separate days that were at least 7 days apart. On one day, participants ingested the balanced amino-acid drink, and on the other day they ingested a Trp-depleted drink. The order was balanced between participants. The order of the TRP - and TRP + session was determined before the start of the experiment by the pharmacist and randomized across all subject numbers, independent of group membership. The experimenters were blind to this randomization and were given the mixtures on the basis of a participant and session number. The order was balanced across all sets of mixtures, but due to selective dropout the number of participants receiving the TRP + or TRP - mixture first differed. Participants were trained on a separate day, on which they received instructions for the tasks and performed two blocks of the task in a dummy scanner. On the testing days, the participants arrived between 0900 and 1300 hours, and were scanned between 1400 and 1800 hours. Participants were instructed to refrain from drinking alcohol on the night before the test day and they received a protein-poor breakfast package, which they could eat on the morning of the testing day. After arrival in the laboratory, the participants first completed the questionnaires (see Subjective measures session), and subsequently blood was drawn. Directly after that, they ingested the amino-acid drink, after which they relaxed for approximately $5 \mathrm{~h}$. During this 5-h break, the participants could drink beverages without caffeine, and could eat protein-free food. Right before the scan session, the participants completed a new set of questionnaires and blood was drawn. In the scanner, the participants performed two blocks of the faces task which lasted about 13 min each. A structural brain scan was made between the two task blocks.

\section{Amino-Acid Mixture}

The contents of the two amino-acid mixtures were based on the proportions described by Young et al (1985). The balanced mixture $(78 \mathrm{~g})$ consisted of $4.1 \mathrm{~g}$ L-alanine, $3.7 \mathrm{~g}$ L-arginine, $2.0 \mathrm{~g}$ L-cysteine, $2.4 \mathrm{~g}$ L-glycine, $2.4 \mathrm{~g}$ L-histidine,
$6 \mathrm{~g}$ L-isoleucine, $10.1 \mathrm{~g}$ L-leucine, $6.7 \mathrm{~g}$ L-lysine monohydrochloride, $2.3 \mathrm{~g}$ methionine, $4.3 \mathrm{~g} \mathrm{~L}-$ phenylalanine, $9.2 \mathrm{~g} \mathrm{~L}-$ proline, $5.2 \mathrm{~g}$ L-serine, $4.9 \mathrm{~g}$ L-threonine, $5.2 \mathrm{~g}$ L-tyrosine, $6.7 \mathrm{~g}$ L-valine, and $3.0 \mathrm{~g} \mathrm{~L}$-Trp. For the Trp-depleted mixture $(75 \mathrm{~g})$, the $3.0 \mathrm{~g}$ L-tryptophan was excluded. Tap water $(200 \mathrm{ml})$ was added to make the mixtures drinkable.

\section{Subjective Measures}

A visual analog version of the shortened Profile of Mood States scale (POMS; McNair et al, 1988) was used to assess mood. The POMS has been shown to be sensitive to detect ATD-induced mood changes in FH + subjects (eg Klaassen et al, 1999). The questionnaire consists of 32 bipolar sets of adjectives, which measure five mood dimensions (anger, depression, fatigue, tension and vigor), which were scored on a 10-point scale. A separate questionnaire was used to assess bodily complaints. Finally, participants completed the translated version of the BIS/BAS scales (Carver and White, 1994) after completion of the whole study. These scales were used to assess the sensitivity of the Behavioral Inhibition System and the behavioral activation system McNaughton and Gray (2000). BIS scores have been found to modulate ATD-induced BOLD responses in a facial emotion perception task (Cools et al, 2005, see Introduction).

\section{Biochemical Measures}

Blood samples $(10 \mathrm{ml})$ were taken to determine plasma amino-acid levels. The blood was centrifuged within $30 \mathrm{~min}$ at $4^{\circ} \mathrm{C}$, at 4000 r.p.m. for $10 \mathrm{~min}$. After this, $100 \mathrm{ml}$ plasma was mixed with $4 \mathrm{mg}$ sulfasalicyl acid and frozen at $-80^{\circ} \mathrm{C}$ until analysis (van Eijk et al, 1994). High-performance liquid chromatography (van Eijk et al, 1994) was used to determine plasma amino-acid concentrations. Total Trp and the ratio between Trp and other Large Neutral Amino Acids (LNAAs) are reported.

\section{MRI Acquisition}

Scanning was performed on a 1.5 Tesla Philips ACS-NT scanner (Philips Medical Systems, Best, The Netherlands). The functional scan session consisted of a single shot multiple slice $\mathrm{T}_{2}{ }^{*}$-weighted echo planar imaging (EPI) sequence, sensitive to the BOLD contrast. Scan parameters were $\mathrm{TR}=1.75 \mathrm{~s}, \mathrm{TE}=27 \mathrm{~ms}, 24$ contiguous axial slices, and a voxel size of $3.5 \times 3.5 \times 5 \mathrm{~mm}$. Before the acquisition of the functional images started, two dummy full brain scans were acquired. For anatomical reference, a $3 \mathrm{D} \mathrm{T}_{1}$-weighted fast-field echo scan was acquired with parameters $\mathrm{TR}=11 \mathrm{~ms}, \mathrm{TE}=3.5 \mathrm{~ms}$, and a voxel size of $1 \times 1 \times 1 \mathrm{~mm}$.

\section{Data Analysis}

Biochemical results, subjective measures, and performance were statistically evaluated using SPSS (version 11.0; SPSS Inc., Chicago, IL). Accuracy was used as performance measure and was analyzed with a repeated-measurements General Linear Model (GLM) analysis with emotion (four levels; happiness, disgust, sadness, fear), instruction (direct $v s$ incidental), intensity (intense $v s$ weak), and condition 
$(\mathrm{TRP}+v s \mathrm{TRP}-)$ as within-subjects factors; group $(\mathrm{FH}+$ vs $\mathrm{FH}-$ ) as between-subjects factor; and order (TRP - first vs TRP + first) as covariate. Mood and biochemical results were analyzed with a repeated-measurements GLM with time (T0, right before ingestion of the AA mixture $v s \mathrm{~T} 5,5 \mathrm{~h}$ after ingestion of the mixture) and condition as withinsubjects factors and order as covariate. The imaging data was analyzed using SPM2 (Wellcome Department of Cognitive Neurology, London, UK). First, the time series were corrected for differences in slice acquisition time and realigned. Subsequently, images from the second session were coregistered to the images from the first session, and images were spatially normalized to the standard Montreal Neurological Institute template. Finally, data were smoothed using an $8 \mathrm{~mm}$ Gaussian spatial filter and a high pass temporal filter.

A total of 16 different stimulus events were modeled separately with a simple hemodynamic response function. The four different emotional expressions, two different intensities, and two different task instructions yielded a total of $16(4 \times 2 \times 2)$ separable stimulus events. The hemodynamic response function was modeled to the onset of the stimulus. Contrasts between intense and weak expressions, direct and incidental task instructions, and intensely fearful and happy expressions and their interactions with ATD, as well as the main effect of ATD were computed for each individual participant. Individual contrast images were taken to a second level analysis in which $t$-values were calculated for each voxel treating intersubject variability as a random effect. Instruction and stimulus-related effects and the interaction with ATD were tested using a one-sample $t$-test. Group differences were tested using a two-sample $t$-test. For localizing purposes, the Montreal Neurological Institute coordinates were converted to Talaraich coordinates using a dedicated script (mni2tal.m; available at http://www.mrc-cbu.cam.ac.uk/ Imaging/Common/mnispace.shtml).

First, a whole brain analysis was performed. The results from this analysis were thresholded at $p<0.001$ uncorrected. Results are reported whenever a cluster was significant at $p<0.05$ corrected, or the most significant voxel within a cluster was significant at $p<0.05$ corrected. Second, region of interest (ROI) analyses were performed for areas where significant activation was expected according to our primary hypothesis using the WFU PickAtlas (Maldjian et al, 2003). Due to the key role of the amygdala in emotion, ROI analyses for the left and right amygdala were performed for all contrasts. These ROIs were based on the Talairach Daemon database (Lancaster et al, 2000), which was also used to determine anatomical labels for the specific Talairach coordinates. Based on the study performed by Winston et al (2003), who used exactly the same task in healthy volunteers, specific ROIs were defined for the different contrasts. Based on the center of activation reported in the Winston et al study, corresponding ROIs were selected from the WFU PickAtlas. For the contrast comparing intense expressions with weak expressions, the bilateral fusiform gyrus was selected as ROI. For the contrast comparing the direct and the incidental task, the bilateral anterior and posterior cingulate cortex, right somatosensory cortex, right superior temporal sulcus, and bilateral insula were selected as ROIs.

\section{RESULTS}

\section{Mood}

No significant differences were observed between the TRP + and TRP - session in the baseline ratings of the summed POMS scores. The repeated-measurements GLM analysis with time, condition, and group as factors and order as covariate revealed no significant effects on the summed POMS scores. Due to the specific hypothesis that ATD would affect mood in the $\mathrm{FH}+$ group, we performed a GLM analysis with time and condition as within-subjects factor and order as a covariate for the $\mathrm{FH}+$ group only. This analysis revealed the expected significant interaction between time and condition, $\mathrm{F}(1,11)=6.0, p<0.05$. Follow-up analyses showed a higher summed POMS score at T5 as compared to T0 in the TRP- condition only, and further follow-up analyses in which the subscales were analyzed separately showed a significantly higher score on the depression and the fatigue subscales in the TRPcondition, whereas no effects could be found in the TRP + condition.

\section{Performance}

Accuracy was statistically evaluated with a GLM analysis with emotion, instruction, intensity and condition as within-subjects factors, group as between-subjects factor, and order as covariate and is presented in Table 1. This analysis showed that intensity, $\mathrm{F}(1,21)=10.7, p<0.005$, had a main effect on accuracy. On average, all participants performed more accurately on the faces displaying more intense emotions. Furthermore, interactions were found between intensity and instruction, $\mathrm{F}(1,21)=26.1, p<0.0005$, between instruction and $\operatorname{ATD}, \mathrm{F}(1,21)=9.9, p<0.01$, and between instruction, order, and ATD, $\mathrm{F}(1,21)=9.4, p<0.01$. Follow-up analyses showed that only in the direct task the intensity of the expressed emotion affected accuracy levels. Further follow-up analyses showed that ATD only affected accuracy in the incidental task. However, this effect of ATD could be completely explained by a task order effect. Accuracy was higher in the first session leading to higher scores in the TRP + condition when this was the first session and lower accuracy scores when this was the second session.

A second set of analyses was performed to examine the effects of an ATD-induced change on the depression subscale of the POMS. We used intensity, instruction, and emotion as within-subjects factors, and the ATD-induced mood effect (POMS depression subscale score at T5 minus score at T0) as covariate. These analyses were performed for the TRP - condition and the $\mathrm{FH}+$ group only because only in this condition and in this group a significant effect on mood was found. This analysis revealed the expected main effect of intensity, $\mathrm{F}(1,11)=40.2, p<0.0005$, and two-way interaction between intensity and instruction, $\mathrm{F}(1,11)=167.9, p<0.0005$. Furthermore, a main effect of emotion, $\mathrm{F}(3,9)=3.8, \quad p<0.05$, a two-way interaction between emotion and intensity, $\mathrm{F}(3,9)=28.8, p<0.0005$, and a two-way interaction between instruction and emotion, $\mathrm{F}(3,9)=14.2, p<0.0005$, were found. Most interestingly, the ATD-induced mood effect significantly interacted with 
emotion and intensity, $\mathrm{F}(1,11)=8.4, p<0.01$. Follow-up analyses, in which correlations were computed between the mood effect and performance related to the different stimulus conditions, showed that there was a significant negative correlation between the mood effect and performance on intense sad and fearful faces in the incidental task and weak faces expressing disgust in the incidental task. For these particular face stimuli, stronger effects on mood (higher score on depression subscale of the POMS) were accompanied by less accurate responses.

\section{Imaging}

Overall effects. The overall effect of ATD on brain activation in the facial emotion perception task was evaluated with a contrast in which all stimulus events of the TRP - condition were compared with all stimulus events

Table I Percentage Correctly Judged (SEM) Stimuli in the Different Conditions of the Task

\begin{tabular}{|c|c|c|c|c|}
\hline Accuracy & Happiness & Fear & Sadness & Disgust \\
\hline \multicolumn{5}{|l|}{$F H-(N=11)$} \\
\hline \multicolumn{5}{|l|}{ TRP+ } \\
\hline \multicolumn{5}{|l|}{ Direct } \\
\hline Strong & $86(4)$ & $84(4)$ & $79(5)$ & $8 \mid(5)$ \\
\hline Weak & $67(7)$ & $44(4)$ & $35(3)$ & $42(3)$ \\
\hline \multicolumn{5}{|l|}{ Incidental } \\
\hline Strong & $66(6)$ & $70(4)$ & $64(5)$ & $66(5)$ \\
\hline Weak & $70(5)$ & $68(3)$ & $69(5)$ & $73(5)$ \\
\hline \multicolumn{5}{|l|}{ TRP- } \\
\hline \multicolumn{5}{|l|}{ Direct } \\
\hline Strong & $90(3)$ & $84(4)$ & $80(4)$ & $83(4)$ \\
\hline Weak & $72(5)$ & $46(3)$ & $4 \mid(4)$ & $46(3)$ \\
\hline \multicolumn{5}{|l|}{ Incidental } \\
\hline Strong & $67(4)$ & $67(4)$ & $70(5)$ & $62(5)$ \\
\hline Weak & $65(4)$ & $66(3)$ & $69(6)$ & $66(5)$ \\
\hline \multicolumn{5}{|l|}{$\mathrm{FH}+(\mathrm{N}=13)$} \\
\hline \multicolumn{5}{|l|}{ TRP+ } \\
\hline \multicolumn{5}{|l|}{ Direct } \\
\hline Strong & $94(2)$ & $94(1)$ & $87(2)$ & $88(2)$ \\
\hline Weak & $62(6)$ & $46(5)$ & $40(3)$ & $43(5)$ \\
\hline \multicolumn{5}{|l|}{ Incidental } \\
\hline Strong & $68(4)$ & $63(4)$ & 67 (3) & $64(3)$ \\
\hline Weak & $68(5)$ & $65(5)$ & $67(5)$ & 7I (5) \\
\hline \multicolumn{5}{|l|}{ TRP- } \\
\hline \multicolumn{5}{|l|}{ Direct } \\
\hline Strong & $89(5)$ & $88(5)$ & 81 (3) & $86(3)$ \\
\hline Weak & $64(5)$ & $38(3)$ & $37(4)$ & $47(4)$ \\
\hline \multicolumn{5}{|l|}{ Incidental } \\
\hline Strong & $60(5)$ & $64(6)$ & $68(5)$ & $67(4)$ \\
\hline Weak & $64(5)$ & $68(5)$ & $71(4)$ & $69(5)$ \\
\hline
\end{tabular}

in the TRP + condition. Although the whole brain analysis did not reveal significant effects, ROI analysis revealed that ATD increased activation in the right amygdala $(z=3.09$, $p=0.031$, MNI coordinates $x, y, z=24,0,-20)$. Percent signal change in the right amygdala in response to the different conditions is shown in Figure 1. The effect of group was evaluated with a two-sample $t$-test, which did not reveal significant effects.

Intense vs weak expressions. The BOLD response related to the intensity of the facial expression was examined by comparing intense and weak expressions. The results of these analyses are presented in Table 2 . First we examined the intensity effect independent of depletion condition and group membership. It was found that more intense expressions were accompanied by increased activation in the right amygdala, bilateral fusiform gyrus, right medial frontal gyrus, right middle frontal gyrus, right cuneus, and right insula. ATD and group did not modulate the BOLD response in this contrast.

Direct vs incidental task. The contrast examining the effect of type of task independent of ATD and group membership revealed that the direct task was accompanied by additional activation in the right superior temporal gyrus $(z=3.80$,

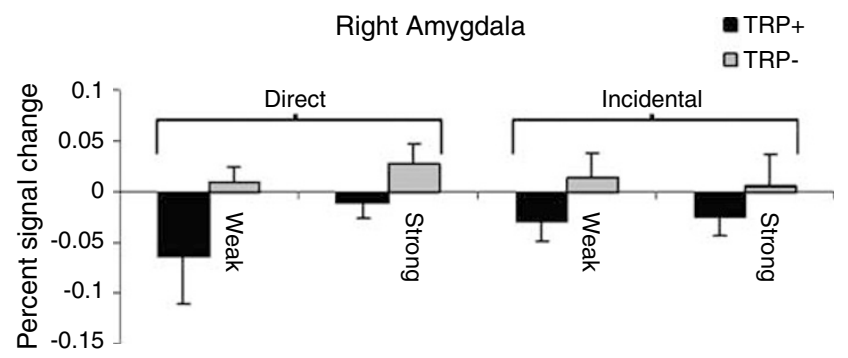

Figure I Percent signal changes as computed by Marsbar (Brett et al 2002) in the right amygdala in response to different facial expressions. Expressions differed with respect to the strength of the expression (weak vs strong) and task instruction (direct vs incidental task). Faces were presented in Trp depletion (TRP-) and the balanced (TRP +) condition.

Table 2 Significant Activation in the Intense-Weak Expressions Contrast

\begin{tabular}{lcccccc}
\hline Region of activation & $\begin{array}{l}\text { Left/ } \\
\text { right }\end{array}$ & $\begin{array}{c}\text { Brodmann } \\
\text { area }\end{array}$ & z-score & $\boldsymbol{x}$ & $\mathbf{y}$ & $\mathbf{z}$ \\
\hline Intensity & & & & & & \\
Medial frontal gyrus & $\mathrm{R}$ & 10 & 4.69 & 16 & 32 & -4 \\
Superior temporal gyrus & $\mathrm{R}$ & 38 & 4.61 & 48 & 10 & -22 \\
Middle frontal gyrus & $\mathrm{R}$ & 10 & 4.61 & 38 & 36 & 20 \\
Fusiform gyrus & $\mathrm{L}$ & 37 & 4.53 & -36 & -62 & -18 \\
Cuneus & $\mathrm{R}$ & 18 & 4.13 & 6 & -76 & 14 \\
Insula & $\mathrm{R}$ & 13 & 4.10 & 36 & 2 & 20 \\
Fusiform gyrus & $\mathrm{R}$ & 19 & 4.01 & 40 & -70 & -20 \\
Amygdala & $\mathrm{R}$ & & $3.18 *$ & 28 & -6 & -22 \\
\hline
\end{tabular}

*Significant in $\mathrm{ROI}$ analysis. 
cluster level $p=0.001$, MNI coordinates $x, y, z=40,-54$, 18 ). ATD and group did not modulate the BOLD response in this contrast.

Fearful vs happy faces. The BOLD response related to the difference between happy and fearful faces was examined by contrasting intense fearful and happy faces. No significant activation was found in this contrast, and group and ATD did not modulate activation in this contrast.

In an additional analysis, we tried to replicate the results of Cools et al (2005) who reported that the BIS score was an important predictor of the effect of ATD on right amygdala activation in a contrast comparing fearful and happy faces. They found this effect using a simple regression analysis. Therefore, a similar regression analysis was performed for all participants with a valid BIS score $(N=19)$ for the contrast in which the modulating effect of ATD on the fearful-happy faces' contrast was examined. The analysis, in which we used the amygdala as an ROI, replicated the findings of Cools et al (2005). Threat sensitivity, as measured by the BIS, significantly correlated with the modulating effect of ATD on brain activation in the right amygdala $(z=2.96, p=0.047$, MNI coordinates $x, y, z=26$, $-4,-14)$. Whole brain analyses did not reveal any significant correlated activation changes in other brain regions.

In a final analysis, we examined whether the ATDinduced mood effects modulated the ATD-induced changes in brain activation in the previously described contrast in which the modulating effect of ATD on the fear-happy contrast was examined. A simple regression analysis was performed with the ATD-induced effect on the depression subscale of the POMS as covariate. As is shown in Figure 2, this analysis revealed that the mood effect significantly correlated with activation in the right amygdala $(z=3.06$, $p=0.047$, MNI coordinates $x, y, z=26,0,-18)$. Whole brain analyses revealed an additional significant effect in the ventromedial prefrontal cortex $(z=4.18$, cluster level $p=0.02$, MNI coordinates of peak voxel $x, y, z=16,60,4)$. This cluster partly overlapped with the rostral part of the anterior cingulate cortex (ACC). Recent findings suggested that activation in the rostral part of the ACC is part of a functional loop including the caudal part of the ACC and the amygdala (see Discussion for details), but that the caudal part of the ACC shows an opposite activation pattern to the amygdala and the rostral part of the ACC. In a final exploratory analysis, we therefore examined whether parts of the ACC correlated negatively with the mood effect in an analysis with the ACC as ROI. This analysis revealed a not significant negative correlation in a cluster in the caudal part of the ACC $(z=3.24$, corrected cluster level $p=0.28$, uncorrected voxel level $p=0.0006$, MNI coordinates of peak voxel $x, y, z=-6,22,28)$.

\section{Biochemical Data}

Blood samples of 21 participants were suitable for biochemical analysis. A paired-samples $t$-test revealed that baseline Trp levels and the plasma ratio Trp/ 2 LNAA did not differ between the TRP + and the TRP - sessions. A repeated-measures GLM with time and condition as within-subjects factors, group as between-subjects factor, and

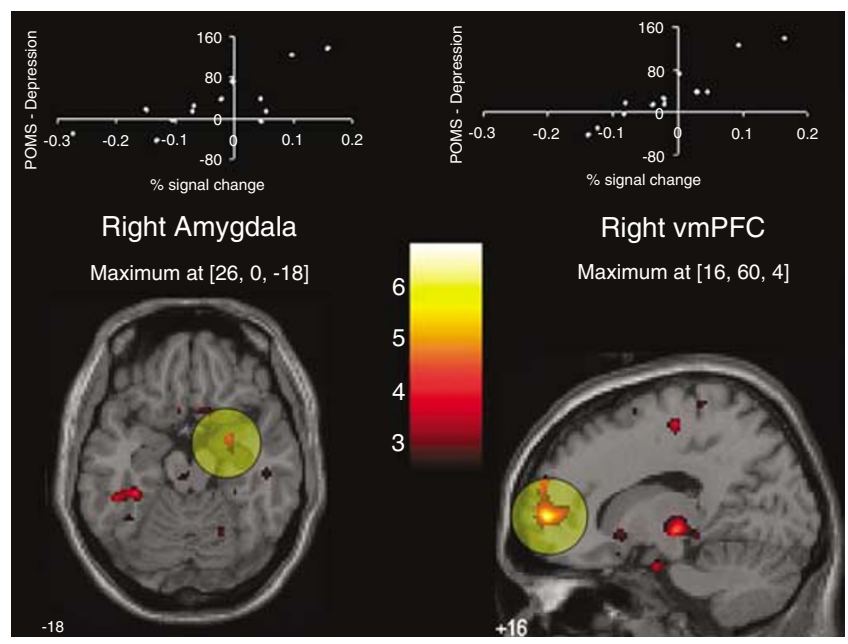

Figure 2 Activation in the right amygdala and right ventromedial prefrontal cortex reflecting a positive correlation between the effect of ATD on the depression subscale of the POMS and brain activation related to the effect of ATD on the difference between intense fearful and happy facial expressions (see text for details). Percent signal changes in the significant clusters as computed by Marsbar (Brett et al, 2002) and the ATD-induced change in the score on depression subscale of the POMS (T5-T0 in the TRP - session) are plotted against each other in the graphs in the top panel.

order as covariate revealed the expected main effects of time, $\mathrm{F}(1,18)=10.75, p<0.005$, and ATD, $\mathrm{F}(1,18)=16.6$, $p<0.005$, and an interaction between these two factors, $\mathrm{F}(1,18)=19.8, p<0.0005$ for Trp levels. On average, Trp levels were higher in the TRP + session and higher at T5. Follow-up analysis showed that Trp levels decreased from T0 to T5 in the TRP - condition (mean \pm SEM; $42 \pm 2 \mu \mathrm{M} / 1$ at T0 vs $10 \pm 1 \mu \mathrm{M} / 1$ at T5) and increased in the TRP + condition (mean \pm SEM; $41 \pm 1 \mu \mathrm{M} / 1$ at T0 vs $132 \pm 15 \mu \mathrm{M} / 1$ at T5). By using the same statistical design for the ratio data, we found a main effect ATD, $\mathrm{F}(1,18)=10.5, p<0.005$, and an two-way interaction between time and ATD, $\mathrm{F}(1,18)=17.4, p<0.005$. Ratios were lower in the TRPcondition and follow-up analyses showed that the ratio decreased from $\mathrm{T} 0$ to $\mathrm{T} 5$ in the TRP - condition (mean \pm SEM $0.12 \pm 0.006$ vs $0.01 \pm 0.002$ ), whereas no difference was found in the TRP + condition (mean \pm SEM $0.11 \pm 0.004 v s$ $0.13 \pm 0.010)$. Group membership and order did not affect Trp levels or plasma ratio $\operatorname{Trp} / \Sigma \mathrm{LNAA}$ values.

\section{DISCUSSION}

The present study examined the effects of ATD on mood, brain activation and performance in a facial emotion perception task in healthy female volunteers with or without a family history of unipolar depression. Pairs of faces displaying a weak or a strong emotion were presented and participants performed a direct or an incidental task. Brain activation in the task was generally in line with the previous study using this paradigm (Winston et al, 2003). ATD lowered mood in the $\mathrm{FH}+$ group and this ATD-induced mood effect was associated with worse performance for negative expressions in the incidental task and with 
stronger amygdala responses to intense fearful faces as compared to happy faces.

ATD differentially affected mood in the $\mathrm{FH}+$ and the $\mathrm{FH}-$ group, which supports our hypothesis. Subjective mood ratings of the $\mathrm{FH}-$ group were not affected by ATD. This is in line with previous studies in which it is generally found that ATD does not affect subjective mood ratings of participants without a personal or family history of affective disorders (eg Schmitt et al, 2000; Evers et al, 2005a, b). Also in line with previous studies is the finding that ATD did affect subjective mood ratings of the $\mathrm{FH}+$ group, that is, this group showed higher summed POMS scores and higher scores on the depression and the fatigue subscales. Mood lowering effects of ATD in FH + participants are reported in a number of studies (Benkelfat et al, 1994; Klassen et al, 1999; Neumeister et al, 2002), whereas only one study reported no effect of a positive family history (Ellenbogen et al, 1999). It should be noted that the exceptionally high dropout rate in the study reporting no effect could have influenced the results.

ATD did not directly affect performance in the present study, which is contrary to our hypothesis. However, it is more or less in line with previous studies, which also reported weak and inconsistent behavioral effects (Hayward et al, 2005; Harmer et al, 2003; Cools et al, 2005). As was suggested in the Introduction, these inconsistencies might be related to the large individual differences in the behavioral response to ATD. In the present study, it was found that performance was modulated by the effect that ATD had on mood. It was found that accuracy scores for fearful or sad expressions in the intense condition or disgust expressions in the weak condition of the incidental task correlated negatively with the ATD-induced change in the score on the depression subscale of the POMS. In other words, a higher depression score was accompanied by less accurate performance. The fact that these effects were only significant in the incidental task might be explained in terms of stronger interference. In the incidental task, participants had to focus on the gender of the face and had to ignore the emotional expression. A stronger effect on mood might have led to a stronger shift in attentional bias towards the more negative emotional content. In this way, the negative expressions that had to be ignored might have had a stronger impact on the decision process and this might have interfered with the main task leading to less accurate responses.

The suggested stronger impact of negative expressions in participants characterized by a stronger effect of ATD on mood was confirmed in the analysis in which correlations were computed between the ATD-induced subjective mood effect and the modulatory effect of ATD on brain activation related to the difference between fearful and happy facial expressions. In this analysis, it was found that a stronger mood effect goes together with stronger right amygdala and right ventromedial prefrontal cortex activation in the contrast comparing intense fearful and happy expressions. It should be noted that we did not find the expected stronger activation of emotion-related areas for the $\mathrm{FH}+$ group as a whole. This is possibly due to variability in the subjective mood response, which was almost absent in some participants and very strong in other participants. In a recent study, Talbot and Cooper (2006) reported that activation in the subgenual part of the anterior cingulate cortex, posterior BA 11 and BA 47, caudate nucleus, and ventral striatum correlated significantly with the ATDinduced mood change. These areas did not overlap with our significantly correlating areas. A possible explanation for this difference might be that Talbot and Cooper examined resting state activation, whereas the present study examined activation differences between fearful and happy faces. In the present design, it was impossible to quantify resting state brain activation. Both the amygdala and the ventromedial prefrontal cortex have been implicated in the processing of emotional stimuli. For instance, Taylor et al (2003) showed that strong amygdala responses could be found when emotionally salient pictures were passively viewed, whereas the ventromedial cortex was more active when the pleasantness of these pictures had to be rated. On the basis of a picture viewing study, Grimm et al (2006) argued that activation in the ventromedial prefrontal cortex is especially sensitive to the valence of the emotional stimulus.

An additional finding was that we replicated the findings of Cools et al (2005), who reported a threat sensitivitydependent effect of ATD on the right amygdala response to fearful faces in healthy males. The participants with higher threat sensitivity scores showed stronger amygdala responses in the TRP - condition. This replication shows that this effect appears to be independent of the gender of the participants, because in the present study only healthy female volunteers were included, whereas Cools et al included only healthy males. This finding was explained in terms of a stronger sensitivity of brain areas involved in the processing of fear stimuli in individuals with a high BIS score, which is possibly mediated by the 5 -HT system. The finding that especially the amygdala is responsive to serotonergic manipulations is in line with a number of previous studies reporting modified amygdala responses as a result of 5-HT manipulations (eg Sheline et al, 2001; Fu et al, 2004; Del-Ben et al, 2005).

Besides the earlier discussed mood and personality related effects of ATD on brain activation, ATD also directly influenced brain activation. ATD generally increased right amygdala activation in the contrast comparing all stimulus events of the TRP- condition with all stimulus events in the TRP + condition. The effects of ATD on amygdala activation are in line with findings of several other studies reporting sensitivity of the amygdala to serotonergic manipulations (eg Sheline et al, 2001; Fu et al, 2004; Del-Ben et al, 2005). These findings are also in line with studies reporting an enhanced amygdala response to fearful stimuli in carriers of the short allele variant of a polymorphism in the promoter region of the 5-HT transporter gene (eg Hariri et al, 2002), who are characterized by a lowered 5-HT metabolism. This enhanced amygdala response is possibly related to reduced inhibition by the caudal or supragenual part of the anterior cingulate cortex (cACC; Pezawas et al, 2005). The amygdala appears to be part of a functional loop in which the amygdala projects to the subgenual or rostral part of the anterior cingulate cortex (rACC; Stefanacci and Amaral, 2002). The rACC has excitatory connections with the cACC that inhibits activation in the amygdala. It has been suggested that this loop is involved in fear extinction (eg Sotres-Bayon 
et al, 2006) and that especially the perigenual (supra- and subgenual parts combined) part of the ACC is strongly dependent on 5-HT metabolism (Varnas et al, 2004). Our findings of stronger amygdala activation going together with lower 5-HT availability are clearly compatible with the existence of such a loop. Moreover, the described enhanced activation of the ventromedial prefrontal cortex could also be seen as compatible. Part of this cluster overlapped with the rACC, and due to the strong projections from the amygdala to the rACC, it would be expected that activation in this area would go together with amygdala activation. Finally, an exploratory analysis of the activation in the cACC showed that there was a trend towards a correlation between activation in this area and mood that was opposite to the correlations found in the amygdala and the rACC. This is also in line with the proposed inhibitory connections between the cACC and the amygdala.

Some caution is warranted as the participants in the present study were all female and relatively young. Previous research (eg Harmer et al, 2003) has shown that gender is an important factor in determining the effects of ATD. Furthermore, the relatively young age of the participants means that some of them have an increased risk to develop depression. Future studies should take these factors into account.

In conclusion, this study provided more evidence for the variable effects of ATD on mood, behavior, and brain activation and for a number of key factors determining the precise effects of ATD. First, genetic factors play an important role, that is, people with specific genetic risk profiles such as the $\mathrm{FH}+$ group in the present study are more vulnerable to the mood lowering effects of ATD and show different brain responses to ATD. Furthermore, personality traits that do or do not depend on this genetic risk profile play a key role in determining the brain response to ATD. Finally, the mood lowering effect of ATD is important in determining the behavioral and neural responses to ATD. Future studies using ATD should take these factors into account and in this way they might shed more light on the complex interplay between environmental and genetic factors determining the effects of ATD on mood, behavior, and brain activation.

\section{ACKNOWLEDGEMENTS}

We thank Christian Röder for his helpful comments on earlier versions of this manuscript, Joel Winston for providing the stimulus material, and Jeroen van Deursen, Gabry Mies, and Frouke Nijhuis for their help in collecting the data. This study was supported by a TOP grant (912-02050) from ZonMW-NWO and a grant from the Dutch Brain Foundation (Hersenstichting, 11F03(2).41).

\section{REFERENCES}

Benkelfat C, Ellenbogen MA, Dean P, Palmour RM, Young SN (1994). Mood-lowering effect of tryptophan depletion. Enhanced susceptibility in young men at genetic risk for major affective disorders. Arch Gen Psychiatry 51: 687-697.

Bhagwagar Z, Cowen PJ, Goodwin GM, Harmer CJ (2004). Normalization of enhanced fear recognition by acute SSRI treatment in subjects with a previous history of depression. $A m J$ Psychiatry 161: 166-168.

Booij L, Van der Does W, Benkelfat C, Bremner JD, Cowen PJ, Fava $M$ et al (2002). Predictors of mood response to acute tryptophan depletion. A reanalysis. Neuropsychopharmacology 27: 852-861.

Brett M, Anton J-L, Valabregue R, Poline J-B (2002). Region of interest analysis using an SPM toolbox [abstract]. Presented at the Eighth International Conference of Functional Mapping of the Human Brain, June 2-6, 2002, Sendai, Japan. Available on CD-ROM in Neurolmage, Vol. 16, No. 2.

Carpenter LL, Anderson GM, Pelton GH, Gudin JA, Kirwin PD, Price LH et al (1998). Tryptophan depletion during continuous CSF sampling in healthy human subjects. Neuropsychopharmacology 19: 26-35.

Carver CS, White TL (1994). Behavioral inhibition, behavioral activation, and affective responses to impending reward and punishment: the BIS/BAS scales. J Pers Soc Psychol 67: 319-333.

Cools R, Calder AJ, Lawrence AD, Clark L, Bullmore E, Robbins TW (2005). Individual differences in threat sensitivity predict serotonergic modulation of amygdala response to fearful faces. Psychopharmacology (Berl) 180: 670-679.

Del-Ben CM, Deakin JF, McKie S, Delvai NA, Williams SR, Elliott R et al (2005). The effect of citalopram pretreatment on neuronal responses to neuropsychological tasks in normal volunteers: an FMRI study. Neuropsychopharmacology 30: 1724-1734.

Ekman P, Friesen WV (1978). Facial Action Coding System. Consulting Psychologists Press: Palo Alto (CA).

Ellenbogen MA, Young SN, Dean P, Palmour RM, Benkelfat C (1999). Acute tryptophan depletion in healthy young women with a family history of major affective disorder. Psychol Med 29: $35-46$.

Evers EA, Cools R, Clark L, van der Veen FM, Jolles J, Sahakian BJ et al (2005a). Serotonergic modulation of prefrontal cortex during negative feedback in probabilistic reversal learning. Neuropsychopharmacology 30: 1138-1147.

Evers EA, Tillie DE, van der Veen FM, Lieben CK, Jolles J, Deutz $\mathrm{NE}$ et al (2005b). Effects of a novel method of acute tryptophan depletion on plasma tryptophan and cognitive performance in healthy volunteers. Psychopharmacology (Berl) 178: 92-99.

Fu CH, Williams SC, Cleare AJ, Brammer MJ, Walsh ND, Kim J et al (2004). Attenuation of the neural response to sad faces in major depression by antidepressant treatment: a prospective, event-related functional magnetic resonance imaging study. Arch Gen Psychiatry 61: 877-889.

Grimm S, Schmidt CF, Bermpohl F, Heinzel A, Dahlem Y, Wyss M et al (2006). Segregated neural representation of distinct emotion dimensions in the prefrontal cortex - an fMRI study. Neuroimage 30: 325-340.

Gur RC, Erwin RJ, Gur RE, Zwil AS, Heimberg C, Kraemer HC (1992). Facial emotion discrimination: II. Behavioral findings in depression. Psychiatry Res 42: 241-251.

Hariri AR, Mattay VS, Tessitore A, Kolachana B, Fera F, Goldman $\mathrm{D}$ et al (2002). Serotonin transporter genetic variation and the response of the human amygdala. Science 297: 400-403.

Harmer CJ, Rogers RD, Tunbridge E, Cowen PJ, Goodwin GM (2003). Tryptophan depletion decreases the recognition of fear in female volunteers. Psychopharmacology (Berl) 167: 411-417.

Hayward G, Goodwin GM, Cowen PJ, Harmer CJ (2005). Low-dose tryptophan depletion in recovered depressed patients induces changes in cognitive processing without depressive symptoms. Biol Psychiatry 57: 517-524.

Klaassen T, Riedel WJ, van Someren A, Deutz NE, Honig A, van Praag HM (1999). Mood effects of 24-hour tryptophan depletion in healthy first-degree relatives of patients with affective disorders. Biol Psychiatry 46: 489-497.

Lacasse JR, Leo J (2005). Serotonin and depression: a disconnect between the advertisements and the scientific literature. PLoS Med 2: e392. 
Lancaster JL, Woldorff MG, Parsons LM, Liotti M, Freitas CS, Rainey L et al (2000). Automated Talairach atlas labels for functional brain mapping. Hum Brain Mapp 10: $120-131$.

Lucki I (1998). The spectrum of behaviors influenced by serotonin. Biol Psychiatry 44: 151-162.

Maes M, Meltzer HY (1995). The serotonin hypothesis of major depression. In: Bloom FE, Kupfer DJ (eds). Psychopharmacology: the Fourth Generation of Progress. Raven Press: New York. pp 933-944.

Maldjian JA, Laurienti PJ, Kraft RA, Burdette JH (2003). An automated method for neuroanatomic and cytoarchitectonic atlas-based interrogation of fMRI data sets. Neuroimage 19: 1233-1239.

McNair DM, Lorr M, Droppelman LF (1988). Manual for the Profile of Mood States. Educational and Industrial Testing Service: San Diego, California.

McNaughton N, Gray JA (2000). Anxiolytic action on the behavioural inhibition system implies multiple types of arousal contribute to anxiety. J Affect Disord 61: 161-176.

Neumeister A, Konstantinidis A, Stastny J, Schwarz MJ, Vitouch O, Willeit $\mathrm{M}$ et al (2002). Association between serotonin transporter gene promoter polymorphism (5HTTLPR) and behavioral responses to tryptophan depletion in healthy women with and without family history of depression. Arch Gen Psychiatry 59: 613-620.

Neumeister A, Nugent AC, Waldeck T, Geraci M, Schwarz M, Bonne $\mathrm{O}$ et al (2004). Neural and behavioral responses to tryptophan depletion in unmedicated patients with remitted major depressive disorder and controls. Arch Gen Psychiatry 61: 765-773.

Pezawas L, Meyer-Lindenberg A, Drabant EM, Verchinski BA, Munoz KE, Kolachana BS et al (2005). 5-HTTLPR polymorphism impacts human cingulated-amygdala interactions: a genetic susceptibility mechanism for depression. Nat Neurosci 8: 828-834.

Schatzberg AF, Garlow SJ, Nemeroff CB (2002). Molecular and cellular mechanisms in depression. In: Davis KL, Charney D, Coyle JT, Nemerhoff C (eds). Neuropsychopharmacology, The
Fifth Generation in Progress. Lippincott Williams \& Wilkins, Philadelphia.

Schmitt JA, Jorissen BL, Sobczak S, van Boxtel MP, Hogervorst E, Deutz NE et al (2000). Tryptophan depletion impairs memory consolidation but improves focussed attention in healthy young volunteers. J Psychopharmacol 14: 21-29.

Sheline YI, Barch DM, Donnelly JM, Ollinger JM, Snyder AZ, Mintun MA (2001). Increased amygdala response to masked emotional faces in depressed subjects resolves with antidepressant treatment: an fMRI study. Biol Psychiatry 50: 651-658.

Sotres-Bayon F, Cain CK, Ledoux JE (2006). Brain mechanisms of fear extinction: historical perspectives on the contribution of prefrontal cortex. Biol Psychiatry 60: 329-336.

Stefanacci L, Amaral DG (2002). Some observations on cortical inputs to the macaque monkey amygdala: an anterograde tracing study. J Comp Neurol 451: 301-323.

Surguladze SA, Young AW, Senior C, Brebion G, Travis MJ, Phillips ML (2004). Recognition accuracy and response bias to happy and sad facial expressions in patients with major depression. Neuropsychology 18: 212-218.

Talbot PS, Cooper SJ (2006). Anterior cingulate and subgenual prefrontal blood flow changes following tryptophan depletion in healthy males. Neuropsychopharmacology 31: 1757-1767.

Taylor SF, Phan KL, Decker LR, Liberzon I (2003). Subjective rating of emotionally salient stimuli modulates neural activity. Neuroimage 18: 650-659.

Van Eijk HM, Rooyakkers DR, Deutz NE (1994). Rapid routine determination of amino acids in plasma by high-performance liquid chromatography with a $2-3$ microns Spherisorb ODS II column. J Chromatogr 620: 143-148.

Varnas K, Halldin C, Hall H (2004). Autoradiographic distribution of serotonin transporters and receptor subtypes in human brain. Hum Brain Mapp 22: 246-260.

Winston JS, O'Doherty J, Dolan RJ (2003). Common and distinct neural responses during direct and incidental processing of multiple facial emotions. Neuroimage 20: 84-97.

Young SN, Smith SE, Phli PO, Ervin FR (1985). Tryptophan depletion causes a rapid lowering of mood in normal males. Psychopharmacology (Berl) 87: 173-177. 